\title{
Análisis de resultados de aprovechamiento e interacción en alumnos de nivel superior al utilizar una aplicación móvil de aprendizaje colaborativo
}

\author{
Analysis of results of use and interaction in higher level students \\ when using a mobile collaborative learning application
}

\author{
Miguel Ángel Couoh Novelo
}

Nelly Sarahi Martin Interian

\begin{abstract}
RESUMEN
Las tecnologías de la información y la comunicación adquieren cada vez más importancia en el ámbito educativo. En este estudio se pretende analizar los resultados de aprovechamiento e interacción de los estudiantes en una institución de educación superior al realizar actividades de aprendizaje colaborativo efectuando un contraste entre el trabajo virtual y el presencial, para verificar la factibilidad de utilizar una aplicación móvil como recurso de aprendizaje colaborativo. Se aplicó una encuesta con respuesta cerrada a 80 alumnos de la carrera de Ingeniería Informática en diferentes semestres y asignaturas. Los resultados demuestran que el uso de nuevos métodos de aprendizaje virtual con alumnos de nivel superior fue un éxito, la aplicación cumplió con las expectativas de mejorar su aprovechamiento de acuerdo con la asignatura, así como interactuar de forma colaborativa para tener mejores resultados. En comparativa entre ambas modalidades, los resultados del trabajo virtual con la aplicación móvil fueron superiores al trabajo manual en rendimiento e interacción.
\end{abstract}

Palabras clave: ambiente educacional, enseñanza superior, rendimiento escolar.

\section{Abstract}

Information and communication technologies are becoming increasingly important in education. This study aims to analyze the results of the use and interaction of students in a higher education institution when carrying out collaborative learning activities by contrasting between virtual and face-to-face work, to verify the feasibility of using a mobile application as a collaborative learning resource. A closed response survey was applied to 80 students of the Computer Engineering career in different semesters and subjects. The results show that the use of new virtual learning methods with higher-level students was a success, the application met the expectations of improving their performance according to the subject, as well as interacting collaboratively to have better results. In comparison between both modalities, the results of virtual work with the mobile application were superior than manual work in performance and interaction.

Keywords: educational environment, higher education, academic achievement. 


\section{INTRODUCCIÓN}

En la actualidad muchas instituciones dedicadas a la educación siguen con el método de aprendizaje en el cual el docente es la figura principal en el aula que se encarga de transmitir sus conocimientos a los estudiantes, quienes adoptan un papel pasivo en las aulas, lo que no permite que tengan la iniciativa de indagar sobre algún tema en específico, de aportar y analizar ideas, o trabajar en equipo.

Lo anterior plantea dificultades para los alumnos, debido a que no obtienen el aprendizaje ni las competencias planteadas inicialmente en los planes de estudio. Por lo tanto, se requiere de metodologías alternativas que incorporen estrategias como el aprendizaje colaborativo y el aprendizaje basado en problemas que a su vez permitan generar un aprendizaje significativo en los alumnos.

Los dispositivos electrónicos en los últimos años se han integrado cada vez más a la educación. Tal como lo menciona la UNESCO (2013), la educación y la tecnología pueden y deben evolucionar en paralelo y apoyarse mutuamente para beneficio de los participantes en el proceso educativo.

Por lo tanto, el objetivo general y los objetivos específicos del presente estudio se enlistan a continuación:

- Objetivo general: Analizar la factibilidad de utilizar una aplicación móvil como recurso de aprendizaje colaborativo, contrastando los resultados de aprovechamiento e interacción en el trabajo virtual con el presencial en una institución de educación superior.

- Objetivos específicos:

1. Identificar la percepción que tienen los estudiantes del TecNM, Campus Tizimín, sobre el aprendizaje móvil.

2. Identificar el sistema operativo factible para realizar la aplicación de acuerdo con los estudiantes del TecNM, Campus Tizimín.

3. Evaluar la aceptabilidad de la aplicación móvil colaborativa de acuerdo con los estudiantes del TecNM, Campus Tizimín.

\footnotetext{
Miguel Ángel Couoh Novelo. Profesor-investigador y jefe del Departamento de Ciencias Básicas del Tecnológico Nacional de México, Campus Tizimín, Yucatán. Es doctor en Educación, con maestría y licenciatura en Ciencias de la Computación. Tiene el reconocimiento al perfil Prodep. Entre sus publicaciones recientes se encuentra el artículo "Evaluación de usabilidad en herramientas de aprendizaje colaborativo en dispositivos móviles para ambientes virtuales educativos". Ha realizado proyectos de investigación relacionados con ambientes virtuales de enseñanza y aprendizaje, aplicaciones móviles y minería de datos. Líder de proyectos de investigación. Correo electrónico: miguelcouoh@gmail.com. ID: https://orcid.org/0000-0001-8490-1954.

Nelly Sarahi Martin Interian. Tecnológico Nacional de México, Campus Tizimín, Yucatán. Es egresada de la licenciatura en Educación de la Universidad Autónoma de Yucatán y colabora desde el 2020 en el Departamento de Ciencias Básicas del Tecnológico Nacional de México, Campus Tizimín, en los proyectos de investigación vigentes. Ha participado en el 1er. Congreso Regional de Computación y Tecnologías de la Información con la presentación del artículo "Validación de la usabilidad del pronunciador de lengua maya Taa’ n en el noreste del estado de Yucatán”. Correo electrónico: sarahimartin97@gmail.com. ID: https://orcid.org/0000-0002-2922-6367.
} 
Se realizó un estudio cuantitativo de tipo descriptivo en el cual se administró una encuesta a los alumnos para conocer su opinión sobre el aprendizaje móvil y la factibilidad de incluir una aplicación móvil en sus clases que les permita trabajar de manera colaborativa con sus equipos. También se realizaron sesiones en diferentes grupos de Ingeniería Informática para probar la aplicación móvil realizada.

\section{REVISIÓN DE LA LITERATURA}

Viñals y Cuenca (2016) expresan que el ámbito educativo no ha podido resistirse a incorporar nuevas tecnologías en el aula, por lo que el reto actual es la adecuación del contenido y metodologías de enseñanza en un contexto social cambiante, principalmente porque los jóvenes han tenido mayor acercamiento a los dispositivos tecnológicos en comparación con las generaciones anteriores.

Pacheco (2019) resalta que la influencia de la tecnología en la sociedad se ha vuelto cada vez más significativa, por lo tanto, en el sistema educativo también se ha integrado la tecnología a pesar de que aún hay barreras por superar, sin embargo, el objetivo que se debe lograr es generar la oportunidad de que el alumno interactúe con la tecnología y no se haga dependiente de ella.

La pedagogía actual debería enfocarse en la libertad de los estudiantes para pensar fuera de lo convencional y establecer metodologías de aprendizaje colaborativo que permitan construir y compartir objetivos que den sentido a las TIC en el desarrollo curricular y la formación estudiantil. El aprendizaje colaborativo implica el trabajo en equipo de los propios estudiantes mediante diferentes estrategias, de forma que cada miembro del equipo sea parte de la responsabilidad (García, Basilotta y López, 2014).

En las actividades de enseñanza-aprendizaje, el trabajo colaborativo o cooperativo, términos utilizados indistintamente, conforma uno de los principales elementos. Los proyectos innovadores que usan técnicas de enseñanza-aprendizaje involucran esta modalidad de trabajo en la que el sujeto se forma como persona (Glinz, 2005).

Trabajando colaborativamente los alumnos aprenden el material al cuestionar, explicar, recordar y asistir a los otros durante el proceso ofreciendo retroalimentación y recibiendo recompensas. Son precisamente esas recompensas las que incitan a los estudiantes en el uso de habilidades de razonamiento, a trabajar duro por el éxito y a ayudarse entre ellos (Agarwal y Nagar, 2011).

Tal como expresa Glinz (2005), en el proceso de enseñanza tradicional el aprendizaje se ha tomado como la transmisión de conocimientos; en los tiempos recientes el paradigma ha cambiado sustentado en la transferencia de conocimientos. Los procesos que los alumnos utilizan son de adecuación, como se plantea en la teoría constructivista. La comunicación con los pares abre la percepción de la persona, desarrolla habilidades cognitivas y de trabajo en grupo, respondiendo a las necesidades que se conciben para esta época. 
En la historia de la humanidad trabajar y aprender juntos es algo vastamente difundido, aunque ya a fines del siglo XX surgió el concepto de "aprendizaje colaborativo", transformándose en un tema de estudio en el que se ha teorizado. El constructivismo sociocultural ha servido como marco teórico para este enfoque del aprendizaje que afirma que todo aprendizaje es social y mediado (Osalde, 2015).

$\mathrm{El}$ aprendizaje cooperativo y el aprendizaje colaborativo son enfoques que en su forma son similares; ambos trabajan con grupos de alumnos, no obstante, algunas características, así como el objetivo que persiguen, las estructuras o el rol del profesor los diferencian. El aprendizaje cooperativo tiene fines socio-efectivos, es decir, que los alumnos se ayuden para lograr una meta, en tanto que el aprendizaje colaborativo busca desarrollar habilidades personales y sociales (Osalde, 2015).

Por lo tanto, a pesar de que los términos de "aprendizaje colaborativo" y "aprendizaje cooperativo", "aprendizaje grupal" y en ocasiones "aprendizaje basado en problemas" son usados como sinónimos hay diferencias entre ambos, lo cual es respaldado por múltiples autores, como se menciona a continuación.

Por su parte, Adell y Bernabé (2006) mencionan que en el aprendizaje cooperativo se da una división de tareas para posteriormente integrarlas para la consecución del objetivo, en cambio en el aprendizaje colaborativo se comparte la responsabilidad dándole mayor énfasis al proceso que a la tarea, de tal forma que se construye el conocimiento a través de la colaboración grupal.

El aprendizaje colaborativo no es solo una técnica de aula sino una manera de vivir y tratar con otras personas. Es el aprendiz quien diseña y controla todas las decisiones que giran en torno a su aprendizaje. En cambio, en el aprendizaje cooperativo la situación de aprendizaje es estructurada de tal forma que todos los miembros del grupo buscan alcanzar una meta en común, convirtiendo la experiencia de aprendizaje en un ejercicio mucho más profundo que una mera forma de agrupamiento entre estudiantes (Trujillo, 2015).

Los elementos básicos que requieren para propiciar el aprendizaje colaborativo, según Lucero (2003), son: interdependencia positiva (los miembros deben confiar y necesitarse los unos a los otros), interacción (el contacto permite el seguimiento e intercambio de los miembros), contribución individual (cada miembro debe asumir su tarea), habilidades personales y de grupo (el grupo debe permitir el desarrollo y potencialización de las habilidades personales).

Barberá, Badia y Mominó (2001) definen la interacción como "un conjunto de reacciones interconectadas entre los miembros que participan en un determinado contexto educativo, en el que la actividad cognitiva humana se desarrolla en función de los elementos que determina la naturaleza de ese contexto educativo, en nuestro caso virtual"' (p. 164).

Albán y Calero (2017) expresan que el rendimiento está relacionado con el esfuerzo y capacidad del estudiante o comprende el proceso de aprendizaje, específica- 
mente el producto obtenido por el estudiante. Actualmente el rendimiento académico "se puede considerar como un estimado de lo que un alumno ha aprendido como consecuencia de un proceso de instrucción o formación; es la capacidad del alumno para responder al proceso educativo en función a objetivos o competencias" (p. 215).

Un concepto ampliamente relacionado con el rendimiento académico y en ocasiones confundido es el aprovechamiento, que es definido como "el nivel de conocimientos, habilidades y destrezas que el alumno adquiere durante el proceso enseñanza-aprendizaje” (Osorio, Mejía y Navarro, 2012, p. 138).

Por otra parte se encuentra el concepto de "aprendizaje móvil", conocido como m-learning, que ha tomado más importancia recientemente, concepto relacionado con el aprendizaje por medios electrónicos (e-learning); como menciona Park (2011), el aprendizaje móvil puede entenderse como una evolución del e-learning en un contexto en el que se posibilita al alumnado el aprovechamiento de las ventajas de las tecnologías móviles como soporte al proceso de aprendizaje, ya que es más accesible un dispositivo móvil.

El aprendizaje móvil promete apoyar la enseñanza virtual por medio de nuevos dispositivos portátiles, que fomenten nuevos sistemas de comunicación entre el hombre y la tecnología (López, Miguel y Fernández-Pampillón, 2008), considerando la facilidad de acceso a los dispositivos en la actualidad.

La aparición de los dispositivos móviles, diseñados en un principio para la comunicación, ha introducido un cambio de paradigma en la educación en general y en la educación a distancia en particular. Un instrumento concebido inicialmente para la comunicación se ha reinventado para utilizarse en la enseñanza como una herramienta didáctica, reconceptualizando y recontextualizando términos hasta ahora considerados absolutos, como eran la distancia y la movilidad (Ortíz, Aveiga, Yederman y Salazar, 2016).

Carneiro, Toscano y Díaz (2009) explican que la masificación de dispositivos móviles de última generación ha facilitado el desarrollo y la implantación de metodologías especialmente diseñadas al medio que cuentan con la ventaja y comodidad de adecuarse a las específicas necesidades -en términos de tiempo y lugar- de los aprendientes.

Los desarrolladores de software de m-learning o aprendizaje móvil han puesto a disposición de los educadores aplicaciones de diversas temáticas para que sean utilizadas como apoyo en el proceso de aprendizaje, estando dentro o fuera del aula (Rodríguez y Juárez, 2017).

Es escasa la documentación (guías de diseño, mejores prácticas o estudios científicos) de cómo debería diseñarse una aplicación de m-learning y cómo debería llevarse a cabo la correcta integración de los distintos componentes educativos para que una aplicación cumpla con el objetivo didáctico establecido; por tanto, una combinación de intuición, destreza y suerte ha guiado a los programadores o diseñadores de software al desarrollo de una aplicación nueva [Rodríguez y Juárez, 2017, p. 5]. 


\section{Método}

\section{Contexto}

El Tecnológico Nacional de México, Campus Tizimín, es una institución pública de educación superior fundada hace 45 años, se ubica al oeste de la ciudad de Tizimín, estado de Yucatán, en México. Oferta las carreras de licenciatura en Administración, licenciatura en Biología, ingeniería en Agronomía, ingeniería Informática e ingeniería en Gestión Empresarial, también cuenta con la maestría en Agroecosistemas Sostenibles.

\section{Participantes}

Otzen y Manterola (2017) mencionan que el muestreo aleatorio simple es una técnica de muestreo probabilístico que garantiza que todos los individuos de una población tienen la misma probabilidad de ser elegidos. Está técnica fue la utilizada para obtener la muestra en el presente estudio.

Se consideró la carrera de Ingeniería Informática, ya que cuenta con los conocimientos y experiencia afines con las aplicaciones móviles, plataformas virtuales, así como todos los tópicos relacionados con el proyecto.

A partir de un muestreo aleatorio simple, se seleccionó a 88 alumnos de la carrera antes mencionada. Se obtuvo a través de las encuestas por muestreo, que consiste en extraer una población finita de $\mathrm{N}$ unidades, subpoblaciones de un tamaño fijo de antemano. Si todas las unidades son indistinguibles, el número de muestra de tamaño $\mathrm{n}$ viene dado por:

$$
\left(\begin{array}{l}
N \\
n
\end{array}\right)=\frac{N !}{n !(N-n) !}={ }_{N} C_{n}
$$

$\mathrm{N}=$ total de estudiantes universidad (1150).

$\mathrm{n}=$ tamaño de la muestra (80).

De la población total de 1,150 estudiantes de diferentes carreras se eligió la muestra de 80 alumnos de la carrera de Ingeniería Informática. Se seleccionaron tres diferentes grupos de la especialidad de Informática, los cuales fueron los siguientes: de octavo semestre con la asignatura de Biometría informática, sexto semestre con la asignatura de Desarrollo de aplicaciones web, y con los de segundo semestre con la asignatura de Fundamentos de sistemas de información; se trabajó de acuerdo con la unidad en la cual se encontraban. 


\section{Procedimiento}

El enfoque del diseño de la aplicación fue el de una plataforma virtual, la cual está estructurada con dos vistas, una es la del usuario o del alumno, en ella se muestran algunos de los servicios de la aplicación móvil, por ejemplo, los foros en los que solo los alumnos pueden debatir sobre algún tema específico. La otra vista es la del administrador o docente, en la que se pueden visualizar todos los servicios que la aplicación tiene, decide a qué alumno añadir en cada equipo y de esta manera el docente tendrá la opción de visualizar qué alumnos participan en la actividad que se le fuera asignada. Cabe mencionar que la aplicación cuenta con más servicios, los cuales son fáciles de manejar por lo que a los usuarios no se les complica por el diseño establecido desde el inicio.

Primero se diseñó la arquitectura del sistema según los requerimientos planteados para tener una visión clara de lo que se desarrolló; se elaboraron pantallas y se analizó la funcionalidad de estas para evitar dificultades de funcionamiento para el usuario. Después se codificaron los módulos del sistema especificados en la parte de diseño de sistema y se elaboró el software.

Ya teniendo la aplicación codificada se procedió a realizar pruebas de funcionamiento según los requerimientos planteados al inicio, se comprobó que la aplicación realice todo lo planteado y se compararon los resultados obtenidos para asegurar que arrojaran los resultados correctos. Posteriormente se iniciaron las pruebas con la muestra seleccionada en las asignaturas ya mencionadas. La recopilación de datos se obtuvo a partir de las clases impartidas, de evaluaciones y encuestas realizadas al finalizar cada sesión de clases.

Se utilizaron patrones de comportamiento clasificado por categorías y subcategorías (alta, media, baja), los análisis de las interacciones que se producen tienen por finalidad comprender cómo es el proceso de enseñanza y aprendizaje en un espacio virtual colaborativo y a partir de ello realizar correcciones que permitan mejorar el rendimiento académico de los estudiantes.

Las técnicas utilizadas fueron seleccionadas de acuerdo con los objetivos planteados y el fenómeno a estudiar, las cuales fueron: observación no participante, encuesta, evaluaciones después de cada sesión de clases y patrones de comportamiento para medir el nivel de interacción de los estudiantes con aplicación de aprendizaje virtual colaborativo.

Finalmente, la encuesta fue elaborada con reactivos de opción múltiple y algunas preguntas abiertas, la cual permitió cumplir con su propósito inicial que fue proporcionar información sobre la aplicación; dicha encuesta se administró a los alumnos de diferentes semestres. También se obtuvo información de las observaciones realizadas. 


\section{Análisis de datos}

Para el análisis de esta encuesta se utilizó el programa Statistical Package for the Social Sciences (SPSS). Es un conjunto de herramientas de tratamiento de datos el cual sirve para los análisis estadísticos de datos, es de mucha utilidad por la facilidad que tiene en almacenar información numerosa, que fue recolectada por parte de los estudiantes que cursan la carrera de Ingeniería Informática en una institución encargada de impartir educación superior en el país.

\section{Resultados}

A continuación se presentan los resultados obtenidos en la investigación realizada; cabe resaltar que se obtuvieron de la observación de las sesiones de clase que fueron grabadas para analizarlas posteriormente, las encuestas realizadas a los estudiantes y la información que proporcionó la aplicación móvil.

\section{Primer instrumento aplicado}

Se llevó a cabo la primera sesión, en la cual se les proporcionó una encuesta a todos los participantes. A continuación se presentan los resultados obtenidos de este primer instrumento.

De acuerdo con la encuesta, la mayoría de los alumnos (90\%) utiliza dispositivos móviles con sistema operativo Android, 5\% con Windows y el 5\% restante usa celulares con sistema operativo IOS. Se tiene un total de $90 \%$ de estudiantes que manejan dispositivos con Android, lo cual indica que es factible realizar objetos de aprendizaje móviles colaborativos como recursos de apoyo para ese tipo de sistema operativo móvil.

Con base en la pregunta “¿por qué consideras que sí o por qué consideras que no son eficientes las apps en tus labores educativas?”, el 70\% correspondiente a la mayoría de los estudiantes considera que sería excelente contar con una aplicación móvil en la cual puedan realizar varias actividades de las asignaturas que cursan.

La segunda respuesta que se obtuvo fue acerca de la cantidad de tiempo invertido en un dispositivo móvil; el $41 \%$ interactúa más de seis horas con su dispositivo móvil; el 5\% interactúa poco tiempo con su dispositivo, debido a que no se promueve el uso del dispositivo móvil como estrategia de aprendizaje en los estudiantes, lo cual podría ser de mucha ayuda.

Otra de las cuestiones fue el conocimiento que tenían sobre el concepto de aprendizaje móvil (m-learning), es decir, del uso de dispositivos móviles para el proceso de enseñanza-aprendizaje. Los resultados indican que 67.5\% de los participantes no había escuchado sobre el uso de los dispositivos móviles en el proceso de enseñanzaaprendizaje (aprendizaje móvil), sin embargo, el 32.5\% ha escuchado sobre este tipo de aprendizaje, aunque no lo han utilizado, debido a que no se ha incluido dentro 
del diseño instruccional de las diferentes asignaturas o como un medio de apoyo para las tareas.

Respecto a la siguiente pregunta, " ¿has tenido alguna experiencia personal o por terceras personas sobre la manera en que otros centros educativos han manejado una metodología de aprendizaje móvil?”, el 69\% de los estudiantes no ha experimentado el aprendizaje móvil, esto se atribuye a que no se promueve el uso del dispositivo móvil como herramienta de aprendizaje y por lo tanto no se promueve el aprendizaje colaborativo, lo cual sería de mucha ayuda para los estudiantes.

En la siguiente pregunta que se realizó con el fin de conocer si los alumnos estarían interesados en este tipo de aprendizaje, “ ¿consideras una ventaja el hecho de incluir en tus asignaturas actividades que incorporen el uso de dispositivos móviles como estrategias de aprendizaje?”, se obtuvo que 99\% de los estudiantes están interesados en que sus asignaturas incluyan actividades que promuevan el aprendizaje móvil. El comentario obtenido de parte de los estudiantes se resume de la siguiente manera:

- Facilita la información necesaria para el desarrollo de las actividades en cualquier momento libre.

- Permite accesibilidad a personas ocupadas laboralmente.

- Es más factible llevar siempre un celular que una computadora personal.

- Permitiría avanzar en las tareas de la ingeniería y de la licenciatura.

- Es una herramienta útil para aquellos que no puedan estar frente a una computadora trabajando.

- El aprendizaje móvil posibilita a muchos superarse y seguir adelante en su preparación académica.

La mayoría de los estudiantes no ha considerado realizar proyectos que se relacionen con este tipo de aprendizaje, el 75.5\% no tiene la iniciativa debido a que no han escuchado hablar sobre este tipo de aprendizaje, pero están dispuestos a aprender; el 24.5\% de los estudiantes ha escuchado hablar de este tipo de aprendizaje, el cual consideran que sería bueno, y han realizado proyectos de investigación relacionados con el tema.

\section{Sesiones de clases}

En los siguientes resultados se observa el nivel de aprovechamiento, de igual manera que el nivel de interacción que tuvo cada estudiante. Estos resultados fueron obtenidos por una serie de patrones y variables asignadas de acuerdo con la observación no participante y por medio de las evaluaciones realizadas por cada alumno.

Como introducción a estos resultados, en la figura 1 se puede observar que se trabajó con un total de 80 alumnos, de los cuales el 50\% (correspondiente a 40 alumnos) trabajó de manera manual, es decir, de la manera tradicional en el salón de clases, y el otro 50\% trabajó de manera virtual por medio de una aplicación móvil de aprendizaje colaborativo. 


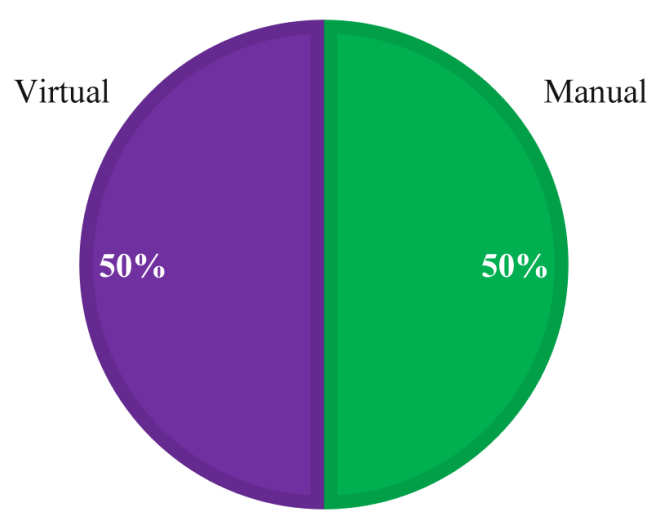

Figura 1. Manera de trabajo de cada alumno.

Fuente: Construcción personal.

Para medir el aprovechamiento de los alumnos participantes se designaron las variables de "aprobó" y "reprobó", cada evaluación aplicada a los alumnos arrojó un resultado; se realizó a todos los participantes, así trabajaran de manera manual como virtual. Cabe mencionar que el examen que se les aplicó fue individual y no de forma grupal, para obtener resultados específicos. Asimismo permitió conocer el nivel de interacción, comparando con la manera de trabajo de cada alumno.

A continuación se presentan los resultados de las cuatro pruebas aplicadas a los estudiantes participantes.

Tabla 1. Análisis del primer examen realizado por cada alumno participante.

\begin{tabular}{llccc} 
& & \multicolumn{2}{c}{ ¿De qué manera trabajó cada estudiante? } & \\
\cline { 2 - 4 } & & Manual & Virtual & Total \\
\hline \multirow{2}{*}{$\begin{array}{l}\text { Primer examen } \\
\text { que se aplicó }\end{array}$} & Aprobados & 25 & 27 & 52 \\
\cline { 2 - 5 } & Reprobados & 15 & 13 & 28 \\
\cline { 2 - 5 } & Total & 40 & 40 & 80 \\
\hline
\end{tabular}

Fuente: Construcción personal.

En la tabla 1 se muestran los resultados de la primera prueba que fue aplicada a los 80 estudiantes participantes; se puede notar que se trabajó de manera igualitaria, es decir que, de los 80 alumnos, se trabajó con 40 de manera virtual y los otros 40 trabajaron de la forma manual como acostumbran en el salón de clases.

Se puede apreciar el porcentaje del nivel de aprovechamiento, el cual se clasificó por las variables (aprobó y reprobó), de esta manera se observa que los alumnos que trabajaron de manera virtual, por medio de una app, tuvieron un porcentaje alto de aprovechamiento, el cual es el siguiente: de los 40 alumnos que trabajaron de forma manual, el 62.5\% aprobó el examen y 37.5\% lo reprobó, mientras que de los alum- 
nos que trabajaron virtualmente por medio de app $67.5 \%$ fueron aprobados y $32.5 \%$ reprobados.

De acuerdo con estos resultados se puede notar que, en este primer examen administrado a los alumnos, los que trabajaron con la app obtuvieron un mayor índice aprobatorio.

Tabla 2. Segundo examen aplicado, de qué manera trabajó cada estudiante.

\begin{tabular}{llccc} 
& & \multicolumn{2}{c}{ ¿De qué manera trabajó cada estudiante? } & \\
\cline { 2 - 4 } & & Manual & Virtual & Total \\
\hline \multirow{2}{*}{$\begin{array}{l}\text { Segundo examen } \\
\text { que se aplicó }\end{array}$} & Aprobados & 26 & 33 & 59 \\
\cline { 2 - 5 } & Reprobados & 14 & 7 & 21 \\
\cline { 2 - 5 } & Total & 40 & 40 & 80 \\
\hline
\end{tabular}

Fuente: Construcción personal.

En la tabla 2 se puede observar que los alumnos que trabajaron con la aplicación móvil obtuvieron un resultado aprobatorio al momento de presentar sus exámenes. Se muestra que el $82.5 \%$ de los alumnos fue aprobado al momento de presentar su examen y el 17.5\% reprobó. Los alumnos que trabajaron de manera manual fueron el 65\% de los aprobados.

De igual manera se observa que 35\% de los participantes en la modalidad manual reprobó el examen, en comparación con los alumnos que realizaron la actividad de manera virtual, por lo tanto, tuvieron menor aprovechamiento ya que el porcentaje de alumnos reprobados fue más alto.

Tabla 3. Tercer examen aplicado y de qué manera trabajó cada estudiante.

\begin{tabular}{llccc} 
& & \multicolumn{2}{c}{ ¿De qué manera trabajó cada estudiante? } & \\
\cline { 2 - 5 } & & Manual & Virtual & Total \\
\hline \multirow{2}{*}{$\begin{array}{l}\text { Tercer examen } \\
\text { que se aplicó }\end{array}$} & Aprobados & 16 & 32 & 48 \\
\cline { 2 - 5 } & Reprobados & 24 & 8 & 35 \\
\cline { 2 - 5 } & Total & 40 & 40 & 80 \\
\hline
\end{tabular}

Fuente: Construcción personal.

En el tercer examen que se les aplicó, cuyos resultados se presentan en la tabla 3 , se puede diferenciar que los alumnos que más aprobaron son los que trabajaron virtualmente, con $80 \%$ de alumnos aprobados y $20 \%$ de alumnos reprobados, a diferencia de los alumnos que trabajaron manualmente, $40 \%$ de estos alumnos fueron aprobados y 60\% reprobados; lo anterior demuestra que los alumnos con mayor aprovechamiento fueron los que trabajaron de forma virtual. 
Tabla 4. Tabla cruzada cuarto examen aplicado de qué manera trabajó cada estudiante.

\begin{tabular}{llccc} 
& & \multicolumn{2}{c}{ ¿De qué manera trabajó cada estudiante? } & \\
\cline { 2 - 5 } & & Manual & Virtual & Total \\
\hline Cuarto examen & Aprobados & 21 & 37 & 58 \\
\cline { 2 - 5 } que se aplicó & Reprobados & 19 & 3 & 22 \\
\cline { 2 - 5 } & Total & 40 & 40 & 80 \\
\hline & & Fuente: Construcción personal.
\end{tabular}

En la tabla 4 se puede apreciar que los alumnos que emplearon el método virtual fueron los que menos reprobaron. En las sesiones de trabajo se pudo observar que los estudiantes que trabajaban de forma manual ya no mostraban interés en los temas, puesto que entre los integrantes del equipo sucedía que solo trabajaba una sola persona y las demás se mostraban desinteresadas, a diferencia de los que trabajaron de manera virtual, ya que al trabajar bajo esta modalidad les interesaba más el tema, de igual manera, se quedaban registradas las veces que cada alumno participaba.

Una de las observaciones al momento de trabajar de manera virtual fue que todo alumno que empleó un dispositivo móvil participaba colaborativamente, es decir que cada integrante aportaba una idea y de esa idea todos colaboraban para realizar la actividad que se había asignado de manera previa, contrario a lo encontrado en los equipos de trabajo manual.

Los equipos que trabajaron de forma manual se encuentran acostumbrados a trabajar con los mismos compañeros en el salón de clases. En algunos casos, de los cuatro integrantes que componían el equipo uno o dos trabajaban y los demás no mostraban interés en realizar la actividad, puesto que se dedicaban a realizar actividades no relacionadas.

De igual forma en algunos equipos se repartían el trabajo y cada uno aportaba la parte que se le asignó, ya que solo les correspondía una parte, no leían ni comprendían todo, por lo tanto, no se observó una verdadera colaboración entre los compañeros.

Tabla 5. Nivel de interacción de qué manera trabajó cada estudiante.

\begin{tabular}{llccc} 
& & \multicolumn{2}{c}{ ¿De qué manera trabajó cada estudiante? } & \\
\cline { 2 - 5 } & & Manual & Virtual & Total \\
\hline \multirow{2}{*}{$\begin{array}{l}\text { Nivel de } \\
\text { interacción }\end{array}$} & Alto & 14 & 32 & 46 \\
\cline { 2 - 5 } & Medio & 25 & 8 & 33 \\
\cline { 2 - 5 } & Bajo & 40 & 40 & 1 \\
\cline { 2 - 5 } & Total & 40 & 80 \\
\hline
\end{tabular}

Fuente: Construcción personal. 
Para medir la interacción de manera virtual en la app en la que realizaron las actividades, se observaron las veces en que cada alumno dialogaba por medio del chat de la aplicación, de igual manera se registraron las ocasiones en que el alumno participaba al momento que realizaba la actividad, y se asignaron las variables alto, medio y bajo dependiendo del recuento de interacción que tuvo cada alumno, como se presenta en la tabla 5 .

Para medir la interacción de forma manual se tomaron en cuenta los videos que fueron grabados en cada sesión de clases, así como los resultados de aprovechamiento que obtuvieron los alumnos; se obtuvo que de 40 alumnos que trabajaron de forma virtual el 80\% obtuvo un nivel de interacción alto y el 20\% un nivel medio, a diferencia de los 40 alumnos que trabajaron de forma manual, solo el 35\% obtuvo un nivel de interacción alto, $62.5 \%$ nivel medio y el 2.5\% nivel bajo.

Finalmente, los resultados de la interacción así como los de aprobación en los alumnos que trabajaron con la aplicación fueron superiores en comparación con los alumnos de la modalidad manual, y además se presentaron menos dificultades al trabajar con la aplicación móvil que en el aula de clases.

\section{Discusión}

A continuación se presenta una descripción de algunos estudios encontrados que guardan similitud con el realizado, debido a la presencia de aplicaciones móviles en el ámbito educativo o la comparativa entre las modalidades educativas presencial o virtual, con la finalidad de verificar si los resultados en este estudio coinciden.

En el presente estudio los resultados arrojan que los alumnos que trabajaron con la modalidad virtual tuvieron mejores resultados en aprovechamiento y nivel de interacción comparados con los alumnos de la modalidad manual; según lo observado, presentaron menos dificultades al finalizar los trabajos asignados, como desinterés por parte de algunos integrantes en la actividad realizada, falta de cooperación entre el equipo y distribución inequitativa del trabajo.

Además, al hacer uso de una aplicación móvil es más fácil hacer un recuento del nivel de interacción, puesto que hay un registro almacenado; de igual forma, hay familiaridad en el uso de los dispositivos y aplicaciones móviles, a diferencia de los alumnos que no interactuaron con la aplicación.

Los hallazgos en el presente estudio destacan las ventajas del aprendizaje móvil sobre el aprendizaje tradicional, puesto que el aprovechamiento de los alumnos que participaron en la modalidad virtual, haciendo uso de la aplicación, fue mayor.

En el 2008, Rivero, Samino y Pérez realizaron una investigación con la finalidad de ofrecer una valoración del grado en que se ven afectadas las calificaciones de los alumnos que optan por complementar la metodología tradicional con un ambiente virtual, que en este caso fue una plataforma respecto a los alumnos que solo utilizan 
el modelo presencial. Los resultados arrojaron que en las asignaturas en que se trabajó con una modalidad mixta las calificaciones tuvieron un incremento, lo cual coincide con lo encontrado en el presente estudio.

Durán y Estay (2012) realizaron un estudio comparativo entre las modalidades presencial y virtual de dieciocho competencias genéricas con estudiantes de licenciatura en Redes de Comunicación; se realizó una investigación de tipo descriptivo; los resultados muestran que las competencias mantienen el mismo nivel de desarrollo en ambas modalidades; el estudio concluye que ambas modalidades permiten el desarrollo de las competencias genéricas según Alfa Tuning, debido a que la modalidad de aprendizaje no es el único factor decisorio en el rendimiento de los estudiantes. Dicho estudio difiere con los resultados encontrados en el presente.

En una investigación realizada por Salgado (2015) se exploraron las experiencias de estudiantes y profesores en un programa de posgrado de modalidad virtual, en cuanto al diálogo que se establece entre estudiantes y docentes. Se llevó a cabo con estudiantes y profesores de las maestrías en Administración de Empresas y Gerencia de Proyectos, de la Facultad de Ciencias Empresariales de la Universidad Latinoamericana de Ciencia y Tecnología, mediante grupos focales, entrevistas y el análisis de un cuestionario institucional de evaluación de los cursos respondidos por los estudiantes. Los resultados de dicha investigación mencionan que los estudiantes demostraron en general un nivel adecuado de satisfacción con los cursos virtuales; se determinó que en los cursos con más alta valoración los estudiantes apuntaron a 17 varios factores claves: el orden o estructura con que el profesor implementa el curso; la retroalimentación que ofrece el docente; la "cordialidad" del profesor, o grado en que motiva, orienta y se identifica con el grupo; así como un nivel óptimo de exigencia académica, entre otros.

Como se puede notar, hay diferencias importantes entre los resultados de los estudios relacionados que se han hecho anteriormente, debido a que en algunos destacan las ventajas del aprendizaje en modalidad virtual y en otros las de la modalidad presencial, esto puede deberse al contexto en que fueron desarrollados, las metodologías utilizadas, los participantes o los objetivos planteados en cada investigación.

\section{Conclusiones}

De acuerdo con los resultados obtenidos, se considera factible utilizar una aplicación móvil como recurso de aprendizaje colaborativo, ya que los resultados de aprovechamiento e interacción en el trabajo virtual fueron superiores a los del trabajo presencial. De igual forma se encontró interés y aceptabilidad por parte de los alumnos, quienes destacaron las ventajas que encuentran.

El objetivo general del presente estudio ha sido comprobado. El uso de ambientes virtuales colaborativos en el salón de clases se considera factible como metodología 
de aprendizaje en el Tecnológico Nacional de México Campus Tizimín, de acuerdo con el contraste de resultados entre ambas modalidades.

Se comprobó que no todos tienen el concepto de m-learning o aprendizaje móvil, sin embargo, no se observó un rechazo o respuesta negativa ante esta modalidad, puesto que los estudiantes tienen una percepción positiva del aprendizaje móvil, y están conscientes de las ventajas y facilidades que puede tener el uso de esta metodología, lo cual puede generar un ambiente propicio al trabajarla en las aulas de la institución.

De igual manera, la mayoría de los encuestados (99\%) considera una opción pertinente incluir estrategias de aprendizaje móvil en la enseñanza de las asignaturas, por lo tanto, la percepción de los estudiantes sobre el aprendizaje móvil es excelente, ya que lo consideran como una buena alternativa.

Respecto al sistema operativo, lo más factible según los resultados encontrados es el uso del sistema Android, ya que la mayoría cuenta con este. Por esa razón la aplicación móvil se realizó con este sistema operativo, considerando que los alumnos no tengan dificultades durante el proceso de instalación y posterior uso, según se demostró durante las pruebas que se realizaron en los salones de clases.

En conclusión, el uso de nuevos métodos de aprendizajes virtuales con alumnos de nivel superior fue un éxito, en este caso la aplicación móvil diseñada cumplió con las expectativas de mejorar su aprovechamiento de acuerdo con su asignatura, así como interactuar de forma colaborativa para obtener mejores resultados.

Como recomendaciones futuras se determinó realizar mejoras en las redes inalámbricas para brindarles a los alumnos y profesores un buen internet y, de igual manera, inculcar a los profesores a no permanecer con el mismo método de aprendizaje como tradicionalmente lo hacen, y se sugirió el uso de nuevos métodos educativos que incluyan innovación para el aprovechamiento en el aprendizaje colaborativo de los alumnos.

Para futuras investigaciones se recomienda continuar con las sesiones de clase en otras carreras del Tecnológico y en diferentes semestres, dividiendo a los alumnos en las dos modalidades para comparar si hay variaciones en los resultados, así como incluir otras técnicas de investigación cualitativas como entrevistas a los alumnos y/o docentes y observaciones.

\section{REFERENCIAS}

Adell, J., y Bernabé, I. (2006). El aprendiraje cooperativo en las Webquests. Recuperado de: https://elbonia.cent.uji. es/jordi/wp-content/uploads/docs/Jornades_WQ_ Adell\&Bernabe.pdf.

Agarwal, R., y Nagar, N. (2011). Cooperative learning. Delhi: Kalpaz Publications.

Albán Obando, J., y Calero Mieles, J. L. (2017). El rendimiento académico: aproximación necesaria a un problema pedagó- gico actual. Revista Conrado, 13(58), 213-220. Recuperado de: http://conrado.ucf.edu.cu/index.php/conrado.

Barberá, E., Badia, A., y Mominó, J. (2001), La incógnita de la educación a distancia. Cuadernos de Educación, 1(3). Recuperado de: https://revistas.um.es/redu/article/ view/11511/11091.

Carneiro, R., Toscano, J. C., y Díaz, T. (2009). Los desafios de las TICS para el cambio educativo. Recuperado de: https:// 
cvrecursosdidacticos.com/web/repository/1363092950_ Coll_Desafios_TIC.pdf.

Durán Rodríguez, R., y Estay-Niculcar, C. (2012). Estudio comparativo sobre competencias genéricas en modalidad presencial y virtual en un curso de pregrado de la Universidad Tecnológica de Panamá. Revista Electrónica Actualidades Investigativas en Educación, 12(2), 1-32. Recuperado de: https:/ / www.redalyc.org/pdf/447/44723437010.pdf.

García, A., Basilotta, V., y López, C. (2014). Las TIC en el aprendizaje colaborativo en el aula de primaria y secundaria. Comunicar, 21(42), 65-74. DOI: https://doi.org/10.3916/ C42-2014-06.

Glinz, P. E. (2005). Un acercamiento al trabajo colaborativo. Revista Iberoamericana de Educación, 36(7), 1-14. DOI: https:/ / doi.org/10.35362/rie3672927.

López Alonso, C., Miguel, E. D., y Fernández-Pampillón, A. (2008). Propuesta de integración de LAMS en el marco conceptual del espacio de aprendizaje socio-constructivista E-Ling. European LAMS conference, Cádiz, España. Recuperado de: https:/ / core.ac.uk/download/pdf/19711566.pdf.

Lucero, M. M. (2003). Entre el trabajo colaborativo y el aprendizaje colaborativo. Revista Iberoamericana de Educación, 33(1), 1-21. Recuperado de: https://rieoei.org/historico/ deloslectores/528Lucero.PDF.

Ortíz, K., Aveiga, H., Yederman, H., y Salazar, H. (2016). Enseñanza y utilización de las nuevas herramientas informáticas en los sistemas educativos, con la implementación de dispositivos inteligentes. Revista Atlante: Cuadernos de Educación y Desarrollo, (70). Recuperado de: http://www.eumed.net/ $\mathrm{rev} /$ atlante/2016/04/dispositivos.html.

Otzen, T., y Manterola, C. (2017). Técnicas de muestreo sobre una población a estudio. International Journal of Morphology, 35(1), 227-232. Recuperado de: https://scielo.conicyt.cl/ scielo.php?pid $=$ S0717-95022017000100037\&script $=$ sci_ abstract.

Osalde, M. (2015). El aprendizaje colaborativo y el aprendizaje cooperativo en el ámbito educativo. Recuperado de: https://unimex. edu.mx/Investigacion/DocInvestigacion/El_aprendizaje_colaborativo_y_el_aprendizaje_cooperativo_en_el_ambito_educativo.pdf.

Osorio García, M. C., Mejía Serafín, L. H., y Navarro Zavaleta, J. A. (2012). Perfil del alumno de éxito en el aprovechamiento escolar de la asignatura de física general: Caso del plantel
Ignacio Ramírez Calzada. Espacios Públicos, 15(35), 134151. Recuperado de: https://www.redalyc.org/articulo. oa?id $=67624803009$.

Pacheco, D. (2019). Artistas en el aula de matemática. Illari, (7), 45-47. Recuperado de: https://revistas.unae.edu.ec/ indeqx.php/illari/article/view/306.

Park, Y. (2011). A pedagogical framework for mobile learning: categorizing educational applications of mobile technologies into four types. International Review of Research in Open and Distance Learning, 12(2), 78-102. Recuperado de: http://www.irrodl.org/index.php/irrodl/article/ view/791.

Rivero, L., Samino, R., y Pérez, E. (2008). Rendimiento académico y modelos virtuales de enseñanza universitaria en Economía de la Empresa. Nuevos retos hacia la globalización docente en el marco del Espacio Europeo de Educación Superior. En J. Pindado y G. Payne (eds.), Estableciendo puentes en una economía global. Escuela Superior de Gestión Comercial y Marketing/ESIC.

Rodríguez, J., y Juárez, J. (2017). Impacto del m-learning en el proceso de aprendizaje: habilidades y conocimiento. Revista Iberoamericana para la Investigación y el Desarrollo Educativo, 8(15), 1-24. Recuperado de: http:/ /www.scielo. org.mx/pdf/ride/v8n15/2007-7467-ride-8-15-00363.pdf.

Salgado García, E. (2015). La enseñanza y el aprendizaje en modalidad virtual desde la experiencia de estudiantes y profesores de posgrado [Tesis de Doctorado]. Universidad Católica de Costa Rica, San José, Costa Rica. Recuperado de: https:/ / www.aacademica.org/edgar.salgado.garcia/2.

Trujillo, C. (2015). Aprendizaje cooperativo e individual en el rendimiento académico en estudiantes universitarios: un meta análisis [Tesis de Doctorado]. Universidad Computense de Madrid, Madrid, España. Recuperado de: https://eprints. ucm.es/id/eprint/30997/1/T36191.pdf.

UNESCO (2013). El futuro del aprendizaje móvil. Implicaciones para la planificación y formulación de políticas. Recuperado de: http:/ / repositorio.minedu.gob.pe/bitstream/handle/20.500.12799/2460/El\%20futuro\%20del\%20aprendizaje $\% 20$ movil.pdf? sequence $=1$ \&isAllowed $=y$.

Viñals Blanco, A., y Cuenca Amigo, J. (2016). El rol del docente en la era digital. Revista Interuniversitaria de Formación del Profesorado, 30(2), 103-114. Recuperado de: https:// www.redalyc.org/articulo.oa?id $=27447325008$.

Cómo citar este artículo:

Couoh Novelo, M. Á., y Martin Interian, N. S. (2021). Análisis de resultados de aprovechamiento e interacción en alumnos de nivel superior al utilizar una aplicación móvil de aprendizaje colaborativo. IE Revista de Investigación Educativa de la REDIECH, 12, e1363. doi: 10.33010/ie_rie_rediech.v12i0.1363. 